\title{
Modelo de cuidado para el desarrollo sostenible en comunidades rurales del Perú
}

\section{Model of care for the sustainable development of rural communities in Peru}

\author{
María Rodríguez \\ Universidad Nacional de Trujillo, Ciudad Universitaria Av. Juan Pablo II s/n -Trujillo
}

DOI: https://doi.org/10.33017/RevECIPeru2013.0010/

\begin{abstract}
Resumen
Estudio descriptivo, exploratorio, multicentrico, cualitativo de corte crítico-dialéctico, cuyo objetivo es proponer un modelo de cuidado para el desarrollo sostenible de comunidades rurales en el Perú. Los escenarios de estudio son tres comunidades rurales de las regiones Amazonas, La Libertad y Moquegua, donde las familias y actores sociales serán entrevistados mediante el Cuestionario Desarrollo Humano Sostenible (DHS) y la Guía de Grupo Focal respectivamente, información que servirá para la elaboración del modelo de cuidado para el desarrollo sostenible en comunidades rurales. Se presenta un avance de la caracterización del desarrollo sostenible de la comunidad rural Dos de Mayo, perteneciente a la región Amazonas, a través de aspectos para el desarrollo sostenible de su comunidad referidos por los actores sociales e indicadores positivos de los determinantes sociales y culturales identificados en las familias: la posición económica de las madres es el comercio $20 \%$ y de los padres, la agricultura $72.5 \%$; tienen vivienda propia $75 \%$, desagüe $85 \%$, agua entubada $97.5 \%$; poseen conocimiento del uso medicinal de las plantas y las usan para tratar sus problemas de salud. El modelo de cuidado pretende partir de las propias capacidades de la comunidad convirtiéndola en la gestora de su desarrollo en forma sostenible.
\end{abstract}

Descriptores: modelo, desarrollo sostenible, comunidad rural

\begin{abstract}
Descriptive study, exploratory, multicenter, qualitative of critical cutting-dialectical, whose objective is to propose a model of care for the sustainable development of rural communities in Peru. The scenarios of study are three rural communities of the regions Amazons, La Libertad and Moquegua, where families and social actors will be interviewed by means of the Questionnaire Sustainable Human Development (SHD) and guide the Focal Group respectively, information that will be used in the preparation of the care model for sustainable development in rural communities. It presents an advance of the characterization of the sustainable development of the rural community Dos de Mayo, pertaining to the region Amazons, through aspects for the sustainable development of the Community referred by positive indicators and social actors of the social and cultural determinants identified in families: the economic position of mothers is the trade $20 \%$ and of the parents, the agriculture $72.5 \%$; they have own house $75 \%$, drain $85 \%$, water tubed $97.5 \%$; they have knowledge of the medicinal use of plants and use them to treat their health problems. The model of care to starting from the capacities of the community into the management of its development in a sustainable manner.
\end{abstract}

Keywords: model, sustainable development, rural community 


\section{Introducción}

El desarrollo de la sociedad, rica o pobre, puede juzgarse a través de la calidad de la salud de su población, cuán equitativamente está distribuida la salud en el espectro social y el grado de protección brindado contra las desventajas como resultado de una enfermedad o daño. Es el resultado de la unión de diversos aspectos corno educación, salud, libertad, participación y equidad, que se plasma en los diversos pueblos, ámbito en el cual el ser humano se desenvuelve y donde se define la suerte de su gestión [1].

El desarrollo sostenible es multidimensional, posee dimensiones en lo ambiental, lo económico, lo social, lo cultural, etc. Mientras que el desarrollo humano sostenible producido por y para la gente, cuyo indicador de éxito es el mejoramiento de los niveles sociales de vida de la gente, sin hipotecar la vida de las generaciones futuras, pone al hombre en el centro, como pro-bien, pro-empleo, pro-naturaleza y pro-equidad [2].

El Perú es un país diverso, donde coexisten muchas formas de entender los procesos de enfermar y morir. Hay muchos sistemas y modelos para generar desarrollo, objeto de esta investigación.

La desigualdad en el desarrollo de los peruanos está reflejada en indicadores como: el $27,2 \%$ de los hogares del área urbana están en el quintil superior de riqueza y $2,7 \%$ en el quintil inferior; en cambio, en el área rural se presentó lo contrario, $60,2 \%$ de los hogares se ubicaban en el quintil inferior y $0,7 \%$ en el quintil superior; analfabetismo total o parcial mayor en el área rural (22,3\%) que en la urbana $(3,2 \%)$, a predominio en la población indígena y femenina [3].

En el año 2011 la población rural del Perú constituía el $26 \%$, según las proyecciones a partir del censo de población del 2007, donde esta era el 28\% [4]. A través de los años ha ido disminuyendo el crecimiento de la población rural en relación a la población total. Sin embargo, las mayores inequidades e indicadores negativos están esta población.

A pesar que en el área rural la tasa de mortalidad infantil descendió de 78 a 26 defunciones entre la ENDES 1991-1992 y ENDES 2011; los mayores niveles de mortalidad infantil se presentaron en la sierra (22 por mil), en la selva (20 por mil) y en el área rural (26 por mil) y la desnutrición crónica afectó en mayor proporción a niñas y niños del área rural (37\%), es decir, 26,9 puntos porcentuales más que en el área urbana (10,1\%) [3].

En el Perú el modelo de respuesta social del estado está centrado en políticas asistencialistas que no generan competencias para el desarrollo y muy por el contrario generan dependencia [5].

El gobierno brinda servicios mínimos para subsistir, a través de los programas sociales, sin rescatar los recursos y capacidades de las comunidades para que generen desarrollo humano sostenible.

El modelo de atención integral de la salud, vigente estimula la prevalencia de los patrones saludables de la cultura biomédica sobre los tradicionales propios de los diferentes pueblos, considerándoles incluso como conductas no saludables y a los agentes de la medicina tradicional como opositores o como ayudantes de actividades sanitarias propias de la cultura biomédica.

Para promover el desarrollo sostenible de una comunidad, implica analizar la población desde sus determinantes inmediatos 0 fenoménicos (ambientales, biológicos, psicosociales, organización de los servicios sanitarios, estilos de vida, condiciones de vida, etc.) desarrollados en el contexto de procesos económico-sociales más generales (estructura económica de la sociedad) y que está en interacción permanente y dialéctica con los niveles supra estructurales de la sociedad (jurídico, político y cultural).

En un modelo de desarrollo comunitario, las competencias individuales y colectivas deben ser aprovechadas para asegurar el bienestar 0 desarrollo como garantía de libertad. Para crear un modelo de cuidado enfocado al desarrollo humano sostenible, con la participación multisectorial articulada entre sí y con los diferentes niveles de gobierno, un enfoque de determinantes sociales ofrece una mejor perspectiva de resultados sostenibles y equitativos [6].

El actual modelo de políticas públicas privilegia el aspecto negativo de las condiciones de vida de la población, los cuales son tomados como factores de riesgo. Las estrategias de salud se enfocan a controlar los factores de riesgo individuales, con enfoque epidemiológico; más no considera el aspecto positivo que el enfoque de determinantes ofrece y ayudaría al desarrollo de las comunidades a partir de las propias capacidades de la población y por tanto al bienestar y desarrollo de la misma. 
Los profesionales de la salud defienden la biomedicina por encima de las prácticas ancestrales de la medicina tradicional, dejando de lado la cosmovisión propia de las familias rurales, la manera como manejan los recursos naturales que se encuentran en su entorno, entre otros aspectos que forman parte de los determinantes culturales.

El interés de la presente investigación es ofrecer un modelo de cuidado orientado a conseguir cambios estructurales y sistémicos "para si" con el fin de conseguir el desarrollo sostenible de las comunidades. Para ello la investigación se planteó como objetivos la caracterización de las formas y manifestaciones de desarrollo en comunidades rurales teniendo en cuenta sus determinantes culturales y sociales; discutir el desarrollo de las comunidades rurales, desde la perspectiva de sus integrantes y finalmente modelar el cuidado comunitario para el desarrollo.

\section{Metodología}

Es descriptiva, exploratoria y multicéntrica. La trayectoria metodológica multicéntrica brindará herramientas para apoyar las decisiones políticas, el aprendizaje social, la responsabilidad de los diferentes niveles de gobierno y de la ciudadanía, la mejora del cuidado de las comunidades rurales y de la gestión pública, la valorización de las capacidades comunales y finalmente formular la propuesta del modelo de cuidado para el desarrollo de las comunidades rurales. El abordaje metodológico usado es cualitativo de corte crítico-histórico dialéctico en la medida que facilita un análisis dual dialectico del problema en estudio desde la perspectiva de los actores sociales y permite la participación de la sociedad para que genere su propio desarrollo [7] [8] [9].

El estudio se está realizando en tres escenarios con características particulares y comunes, comunidades rurales de tres regiones geopolíticas del país ubicadas cada una en la costa, sierra y selva del Perú. Las unidades de análisis son las familias y actores sociales reconocidos en las comunidades rurales ubicadas en las regiones Amazonas, La Libertad y Moquegua.

El muestreo es con informantes estratégicos; en este caso son las familias y los actores sociales de las comunidades rurales en estudio.

\subsection{Procedimiento}

Para la recolección de datos la técnica usada fue la encuesta y como instrumento se usó el Cuestionario de Desarrollo Humano Sostenible (DHS), el que consiste en la interrogación a un número significativo de familias que expresarán su conocimiento, así como experiencias e inquietudes sobre el desarrollo de su comunidad a través de la descripción de sus determinantes sociales y culturales.

El diario de campo y la grabación son dos técnicas que fueron usadas para recolectar información de los actores sociales.

El análisis de las unidades de significado definidas como las centrales del discurso, será realizada en tres etapas: pre-análisis; exploración del material y el tratamiento de los resultados obtenidos; el análisis e interpretación.

Una vez sistematizada la información de las tres comunidades de estudio en base a los determinantes sociales y culturales, se discutirá comparativamente el desarrollo sostenible de las comunidades rurales seleccionadas para el estudio; de su cruzamiento será posible descifrar aspectos que la apariencia no logra revelar y que servirán para la construcción del modelo de cuidado para el desarrollo.

Se hicieron uso de las consideraciones éticas para la investigación: confidencialidad de la información y el anonimato.

\section{Resultados y discusión}

Se presentan algunos avances de la tesis doctoral, respecto al primer objetivo de la investigación: caracterización del desarrollo de las comunidades rurales, teniendo en cuenta los determinantes sociales y culturales de las familias de una de las comunidades rurales consideradas para el estudio, el Centro Poblado Menor Dos de Mayo, ubicado en el distrito de Leymebamba, provincia Chachapoyas, región Amazonas.

La estimación del Desarrollo Sostenible resulta compleja por la naturaleza de sus componentes económico, social, ambiental y cultural [10].

El componente económico del desarrollo sostenible, señala que las sociedades se encaminen por sendas de crecimiento económico, que generen un flujo óptimo de ingresos a la vez que mantienen las existencias básicas de capital (de fabricación humana, humano y natural) e internalizar todos los costos, incluyendo los costos sociales y ambientales 
relacionados con la producción y disposición de los bienes [11].

Dejar de estudiar, de tener su preparación académica en lo individual, pone a la persona en una desventaja muy importante en el mundo laboral, y evidentemente esto va a repercutir en un menor ingreso económico [12], el cual ya es observado en los padres.

Sin embargo, al ser baja la deserción escolar de los hijos (Ver Tabla 1); aleja el fantasma de la incapacidad de encontrar empleo y de generar bajos ingresos conducentes a mayor pobreza y menor desarrollo de la comunidad.

Tabla 1: Caracterización del desarrollo humano sostenible (dimensión económica), a partir de determinantes sociales estructurales de 40 familias de la Comunidad Dos de Mayo, región Amazonas.

\begin{tabular}{|c|c|c|c|}
\hline $\begin{array}{c}\text { Indicador } \\
\text { económico } \\
\text { de } \\
\text { desarrollo } \\
\end{array}$ & Determinantes sociales estructurales & № & $\%$ \\
\hline $\begin{array}{l}\text { Baja } \\
\text { deserción }\end{array}$ & $\begin{array}{l}\text { Abandono de los estudios a nivel } \\
\text { primario }\end{array}$ & 1 & 2.5 \\
\hline escolar & $\begin{array}{l}\text { Abandono de los estudios a nivel } \\
\text { secundario }\end{array}$ & 2 & 5 \\
\hline Posición & Madres comerciantes. & 8 & 20 \\
\hline económica & Madres agricultoras. & 5 & 12.5 \\
\hline determinada & Padres agricultores. & 29 & 72.5 \\
\hline por & Padres ganaderos. & 4 & 10.0 \\
\hline $\begin{array}{l}\text { actividades } \\
\text { potenciales }\end{array}$ & Padres comerciantes. & 2 & 5.0 \\
\hline $\begin{array}{l}\text { Padres y } \\
\text { madres de }\end{array}$ & $\begin{array}{l}\text { Los padres tienen ingreso económico } \\
\text { mayor a S/. } 200.00\end{array}$ & 18 & 45.0 \\
\hline $\begin{array}{l}\text { familia que } \\
\text { trabajan } \\
\text { aportan a la } \\
\text { canasta } \\
\text { familiar. }\end{array}$ & $\begin{array}{l}\text { Las madres con ingreso económico } \\
\text { mayor a S/. } 200.00\end{array}$ & 5 & 12.5 \\
\hline
\end{tabular}

Fuente: Cuestionario DHS

Las actividades económicas predominantes en la que trabajan las madres de familia son el comercio y la agricultura, mientras para los padres de familia lo constituye la agricultura.

La actividad económica representa un medio importante para salir de la pobreza para un número cada vez mayor de personas del medio rural, sobre todo los jóvenes [13]. La población potencial trabaja preponderantemente en la agricultura y en otros sectores como el comercio, manufacturas y construcción [14]. La participación en la economía rural no agrícola, tanto el empleo asalariado no agrícola como el autoempleo, es un elemento cada vez más importante de las estrategias de gestión del riesgo de un gran número de hogares rurales [13].

La capacidad de ciudadanía es otro determinante estructural social, que permite a los miembros de la familia ejercer sus derechos hacia el desarrollo humano sostenible.

Los derechos económicos, sociales y culturales, incluyen los derechos a trabajar; recibir igual salario por igual trabajo; gozar de protección contra los efectos de la enfermedad, la vejez, la muerte, la incapacidad y el desempleo involuntario [12].

Las condiciones para el "despegue" que desencadenarían la transición hacia las fases avanzadas del desarrollo, no sería sólo de carácter económico sino también cultural y social [15].

Los determinantes sociales y culturales ofrecen indicadores que permiten analizar desde un enfoque positivo, el desarrollo sostenible de una comunidad.

Los determinantes sociales pueden ser estructurales: la estructura social, la posición socioeconómica y la clase social, de ellos depende el nivel educativo que influye en la ocupación y ésta última en el ingreso [16].

La educación y el empleo remunerado de las mujeres, son determinantes sociales estructurales del desarrollo sostenible que pueden incidir especialmente en la reducción de las desigualdades de género, elemento central del subdesarrollo en muchos lugares del mundo.

La sostenibilidad social conduce a un mayor acceso e igualdad social, al respeto por los derechos humanos y a mejoras en materia de salud, educación, oportunidades y otros aspectos no monetarios del bienestar [11].

Los determinantes sociales a su vez están influidos por un contexto sociopolítico (tipo de gobierno, las políticas macro-económicas sociales y públicas, la cultura y los valores sociales) y tienen impacto en la equidad en salud y en el bienestar a través de su acción sobre los determinantes intermediarios: circunstancias materiales (condiciones de vida y de trabajo, disponibilidad de alimentos, etc.), comportamientos, factores biológicos, factores psicosociales y el sistema de salud [16].

Las condiciones de vida, determinante social intermediario, están "relacionadas a las condiciones de alimentación, habitación saneamiento, transporte y medio ambiente, entre otros" [17] y con el nivel de desarrollo económico y accesibilidad al desarrollo social acumulado en el respectivo país [18].

Tabla 2: Caracterización del desarrollo humano sostenible (dimensión social), a partir de determinantes sociales estructurales e intermedios 


\begin{tabular}{|c|c|c|c|}
\hline $\begin{array}{l}\text { Indicador } \\
\text { social de } \\
\text { desarrollo }\end{array}$ & Determinantes sociales estructurales & № & $\%$ \\
\hline $\begin{array}{l}\text { Ejercicio de } \\
\text { ciudadanía }\end{array}$ & $\begin{array}{l}\text { Familias con miembros familiares que } \\
\text { cuentan con DNI. } \\
\text { Determinantes sociales intermedios: }\end{array}$ & 33 & 82.5 \\
\hline $\begin{array}{l}\text { Adecuadas } \\
\text { condiciones } \\
\text { de vida }\end{array}$ & $\begin{array}{l}\text { Tienen vivienda propia. } \\
\text { Tienen vivienda con tres ambientes. } \\
\text { Eliminan basura en carro recolector. } \\
\text { Eliminan las excretas en desagüe. } \\
\text { Tienen agua entubada. }\end{array}$ & $\begin{array}{l}30 \\
35 \\
27 \\
34 \\
39\end{array}$ & $\begin{array}{l}75.0 \\
87.5 \\
67.5 \\
85.0 \\
97.5\end{array}$ \\
\hline $\begin{array}{l}\text { Fácil acceso } \\
\text { a los servicios } \\
\text { de salud. }\end{array}$ & $\begin{array}{l}\text { Acuden a Control de gestación. } \\
\text { Acuden a programa de Inmunización. } \\
\text { Acuden a Consulta médica. } \\
\text { Acuden a Control de Crecimiento y } \\
\text { Desarrollo. }\end{array}$ & $\begin{array}{l}31 \\
24 \\
23 \\
18\end{array}$ & $\begin{array}{l}77.5 \\
60.0 \\
57.5 \\
45.0\end{array}$ \\
\hline $\begin{array}{ll}\text { Vida larga } & \text { y } \\
\text { saludable } & \\
\text { Acceso } & \text { a }\end{array}$ & $\begin{array}{l}\text { Tuvieron hijos con peso al nacer > } \\
2500 \text { gr. } \\
\text { Los hijos recibieron alimentación }\end{array}$ & 27 & 67.5 \\
\hline alimentación & $\begin{array}{l}\text { complementaria desde los } 6 \text { meses. } \\
\text { Los hijos recibieron alimentación } \\
\text { complementaria después de los } 7 \\
\text { meses. }\end{array}$ & 29 & 72.5 \\
\hline & Acceden a alimentación balanceada. & 10 & 25.0 \\
\hline $\begin{array}{l}\text { Adecuada } \\
\text { práctica de } \\
\text { actividades }\end{array}$ & $\begin{array}{l}\text { Frecuentemente realizan caminatas. } \\
\text { Frecuentemente padres juegan con } \\
\text { sus hijos. }\end{array}$ & $\begin{array}{l}38 \\
27\end{array}$ & $\begin{array}{l}95.0 \\
67.5\end{array}$ \\
\hline $\begin{array}{l}\text { de la vida } \\
\text { diaria }\end{array}$ & $\begin{array}{l}\text { Frecuentemente realizan paseos. } \\
\text { Asisten a asociaciones de la } \\
\text { comunidad. }\end{array}$ & $\begin{array}{l}21 \\
10\end{array}$ & $\begin{array}{l}52.5 \\
25.0\end{array}$ \\
\hline
\end{tabular}

de 40 familias de la Comunidad Dos de Mayo, región Amazonas.Fuente: Cuestionario DHS.

Un determinante social intermedio positivo es el acceso a una vida larga y saludable, obtenido del factor biológico peso al nacer y es medida según la probabilidad al nacer de no vivir hasta los 40 años [19].

Los bebes que nacen con bajo peso, inferior a los 2500 gramos, tienen hasta tres o cuatro veces más riesgo de morir, durante los primeros meses y años; esta susceptibilidad de morir por infección se mantiene hasta cuando son adultos jóvenes, con riesgo 10 veces más alto que los eutróficos al nacimiento [20].

Los que sobreviven tienen mayor riesgo de padecer enfermedad infecciosa aguda durante la infancia, conllevando a desnutrición y al círculo vicioso infección/desnutrición/infección y a un incremento de desarrollar enfermedades crónicas degenerativas (hipertensión arterial sistémica, diabetes tipo II, obesidad y osteoporosis) durante la edad adulta [21][22]; además de alteraciones del desarrollo mental, problemas del aprendizaje y del lenguaje; secuelas motoras y auditivas y alteraciones de la conducta [23].

Los hijos de los pobladores de Dos de Mayo tienen probabilidades de una vida larga y saludable ya que el $67.5 \%$ nacieron con un peso dentro de los rangos normales (> 2500gr.), recibieron alimentación complementaria adecuada y practican factores psicosociales positivos: realizan caminatas, juegan con sus hijos, paseos con la familia, asisten a asociaciones de la comunidad (Ver Tabla 2).

Los actores sociales realizan diversas propuestas sobre el desarrollo sostenible de su comunidad, tales como el fortalecimiento de capacidades tecnológicas en actividades económicas potenciales como la agricultura, ganadería, comercio, el acceso a nuevos mercados", "capacitación, proyectos productivos" son algunos aspectos que los agentes sociales de la comunidad en estudio, refiere que se debe considerar para el desarrollo económico de la comunidad (Ver Tabla 3).

Tabla 3: Categorías sobre desarrollo sostenible comunal generado de los discursos de los actores sociales de la Comunidad Dos de Mayo. Fuente: Entrevista a acores sociales. 
Es condición necesaria que la magnitud de los recursos disponibles, sobre todo económicos, sean suficientes para la satisfacción de las necesidades de los individuos de una sociedad y que estas actúen como determinantes para un mayor acceso a los servicios de salud, de educación, de alimentación, de vivienda y desde luego a salarios 0 sueldos "decorosos".

Otra propuesta es la formulación de proyectos educativos y económicos que mejoren sus condiciones económicas para cumplir con el derecho a percibir un ingreso que asegure una existencia conforme a la dignidad humana; disfrutar de un nivel de vida adecuado que garantice la salud y el bienestar; disponer de descanso y de tiempo libre [12].

Las capacidades son el único medio adecuado para que cada ciudadana y ciudadano aproveche las oportunidades generadas por el crecimiento, con vistas a potenciar el abanico de sus opciones y libertades, o lo que es igual, a aprovechar el potencial de desarrollo humano [24].

Las oportunidades sociales referidas a los sistemas de educación, sanidad, etc., que tiene la sociedad y que influyen en la libertad fundamental del individuo para vivir mejor. Estos servicios son importantes no sólo para la vida privada (como llevar una vida sana y evitar la morbilidad evitable y la muerte prematura), sino también para participar más eficazmente en las actividades económicas y políticas [25].

En la dimensión social los actores sociales proponen fortalecer capacidades para mejorar calidad de vida: en cuanto a los servicios de agua, salud, mejorar las viviendas y cocinas, cambio de fuente de energía, mejoramiento de la institución educativa y que las autoridades cuenten con las capacidades adecuadas para gestionar y brindar mejores servicios a la población.

"La cultura es el cuarto pilar del desarrollo sostenible" ... que promueve un crecimiento económico inclusivo, la equidad social, la sostenibilidad medioambiental", ... "para preservar la identidad y la diversidad, tanto local como globalmente" [26].

Los determinantes culturales considerados para determinar el componente cultural del desarrollo humano sostenible de las familias entrevistadas fueron: frecuencia de consumo alimentario, como consumen el agua, uso de remedios caseros, medicamentos o la asistencia al centro de salud para

\begin{tabular}{|c|c|c|c|}
\hline $\begin{array}{c}\text { Categorías } \\
\text { de } \\
\text { desarrollo }\end{array}$ & Discursos & $\begin{array}{l}\text { Código } \\
\text { /actor } \\
\text { social }\end{array}$ & $\begin{array}{l}\text { Dimensiòn/ } \\
\text { desarrollo } \\
\text { sostenible }\end{array}$ \\
\hline $\begin{array}{l}\text { Acceso a } \\
\text { tecnología } \\
\text { agropecuaria } \\
\text { y mercados }\end{array}$ & $\begin{array}{l}\text { "Debe resolverse el acceso al } \\
\text { mercado, acceso a tecnología, } \\
\text { capacitaciones y asistencia } \\
\text { técnica y nuevos cultivos". } \\
\text { "Capacitación, proyectos } \\
\text { productivos". } \\
\text { "Asesoramiento y acceso a la } \\
\text { tecnología de la producción } \\
\text { agropecuaria, acceso a nuevos } \\
\text { mercados. } \\
\text { "Ayudarles en las actividades de } \\
\text { sustento que ellos realizan como } \\
\text { son la ganadería y la agricultura" }\end{array}$ & $\begin{array}{l}\text { ascdma } \\
02 \\
\text { ascdma } \\
03 \\
\\
\\
\text { ascdma } \\
04\end{array}$ & Económico \\
\hline $\begin{array}{l}\text { Vigilancia } \\
\text { social de la } \\
\text { gestión de la } \\
\text { autoridad }\end{array}$ & $\begin{array}{l}\text { "A la sociedad civil le toca } \\
\text { organizarse y fiscalizar el trabajo } \\
\text { de las autoridades". } \\
\text { "La población organizada debe } \\
\text { acompañar y fiscalizar la gestión } \\
\text { de sus autoridades, de } \\
\text { proyectos y actividades que } \\
\text { mejoren su condición de vida" }\end{array}$ & $\begin{array}{l}\text { ascdma } \\
01 \\
\text { ascdma } \\
06\end{array}$ & Social \\
\hline $\begin{array}{l}\text { Mejora de } \\
\text { calidad de } \\
\text { vida }\end{array}$ & 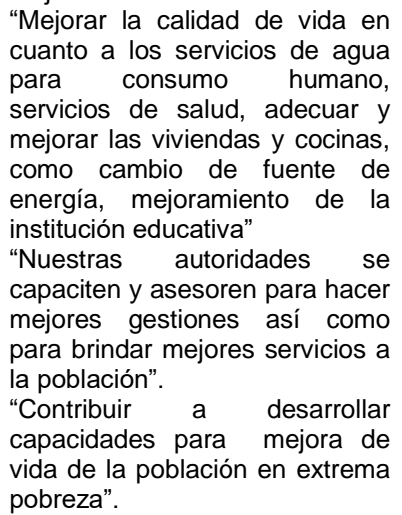 & $\begin{array}{c}\text { ascdma } \\
01\end{array}$ & Social \\
\hline $\begin{array}{l}\text { Actividad de } \\
\text { poco impacto } \\
\text { en el medio } \\
\text { ambiente }\end{array}$ & $\begin{array}{l}\text { "Manejo de la basura y aguas } \\
\text { servidas, organización del } \\
\text { territorio y reforestación de las } \\
\text { cabeceras de los cerros". } \\
\text { "Cambiar el clima soleado para } \\
\text { que no queme los pastos". }\end{array}$ & $\begin{array}{l}\text { ascdma } \\
01 \\
\\
\text { ascdma } \\
07\end{array}$ & Ambiental \\
\hline $\begin{array}{l}\text { Cultura de } \\
\text { funcionalidad } \\
\text { familiar }\end{array}$ & $\begin{array}{l}\text { "Los medios más importantes } \\
\text { son la capacitación en } \\
\text { seguridad alimentaria y } \\
\text { concientización a los padres de } \\
\text { familia para generar una cultura } \\
\text { alimentaria adecuada". } \\
\text { "Que las personas entiendan la } \\
\text { importancia del rol y } \\
\text { compromiso de los padres, } \\
\text { además de organización del } \\
\text { manejo de la casa, de las } \\
\text { responsabilidades para con los } \\
\text { hijos y de la importancia de una } \\
\text { adecuada alimentación". } \\
\text { "Mejorar la calidad de educación } \\
\text { y salud, considerando el cambio } \\
\text { de costumbres y hábitos" } \\
\text { "Los padres se concienticen y se } \\
\text { responsabilicen y pasen la } \\
\text { pensión a alimenticia a sus } \\
\text { hijos". }\end{array}$ & $\begin{array}{l}\text { ascdma } \\
02 \\
\\
\text { ascdma } \\
04\end{array}$ & Cultural \\
\hline
\end{tabular}

tratar a los hijos con diarrea o con tos; para la dimensión ambiental se consideró el uso de gas como combustible para cocinar (Ver Tabla 4).

Tabla 4: Caracterización del desarrollo humano sostenible (dimensiones ambiental y cultural) a partir de determinantes culturales de 40 familias de la 
Comunidad Dos de Mayo, región Amazonas. Fuente: Cuestionario DHS

Entre los grupos sobre los que pesa la pobreza y la exclusión en la selva son: las poblaciones ribereñas, los colonos, los campesinos de montaña y los migrantes. La mayoría de los migrantes provienen de la sierra y $40 \%$ de ellos se distribuyen en la selva alta donde se dedican a la agricultura. Produciendo la andinización de la selva alta [27].

Dos de mayo es una comunidad rural que está ubicada en el Distrito Leymebamba, Provincia Chachapoyas, Región Amazonas, geográficamente está ubicado en Seja de Selva, con regular numero de población foránea, proveniente de Cajamarca y La Libertad, los mismos que traen sus propias costumbres, valores, prácticas culturales y en algunos casos sin documento de identidad que les permita hacer uso de sus derechos, limitando su acceso a servicios básicos educación, salud y trabajo.

En la sierra se cultiva en campo abierto, lo cual no es la costumbre en la Amazonía; los colonos, al no conocer otra forma, talan los árboles para tener un campo abierto. Ello, además de la desertificación, tiene impacto sobre la salud; al cambiar el ecosistema en esa zona se crean las condiciones para la aparición de enfermedades, de allí la importancia de considerar la migración como una de las variables determinantes de la salud de algunos grupos poblacionales [27].

Ante un familiar enfermo lo llevan al centro de salud o compran medicina con prescripción médica. Sin embargo, las familias prefieren usar las plantas medicinales para cuidar la salud de sus miembros, pero no tienen cuidado en reponerlas; el conocimiento ancestral del uso de plantas medicinales en la región amazónica son su capital social y una oportunidad para incrementar sus recursos económicos a partir del manejo adecuado de los recursos naturales de su medio.

La experiencia de Villa El Salvador, donde la clave para la mejora de aspectos fundamentales de la vida de la gente (...) parece hallarse en elementos incluidos en la idea de capital social (...). Los campesinos de los Andes carecían de toda riqueza material, pero llevaban consigo la cultura y la tradición indígenas y una milenaria experiencia histórica de cooperación, trabajo comunal y solidaridad [28].
La combinación de las capacidades individuales y colectivas determina el empoderamiento que habilita a las personas para alcanzar logros concretos; es decir el desarrollo sostenible de su comunidad. Concepto que surgió de su angustia ante las pérdidas de elementos de los ecosistemas, elementos físicos y bióticos de los ecosistemas, ante la desaparición de los bosques naturales, de la

\begin{tabular}{|c|c|c|c|}
\hline $\begin{array}{c}\text { Indicador } \\
\text { ambiental de } \\
\text { desarrollo }\end{array}$ & $\begin{array}{l}\text { Características de desarrollo sostenible } \\
\text { de la comunidad }\end{array}$ & $\mathbf{N}^{\mathbf{o}}$ & $\%$ \\
\hline $\begin{array}{l}\text { Prácticas } \\
\text { ecológicas }\end{array}$ & $\begin{array}{l}\text { Usan el gas como combustible para } \\
\text { cocinar }\end{array}$ & 8 & 20 \\
\hline \multicolumn{4}{|c|}{ Indicador cultural de desarrollo } \\
\hline Adecuadas & Consumen alimentos 3 a 4 veces al día. & 32 & 80 \\
\hline prácticas de & Los niños siempre toman el desayuno. & 32 & 80 \\
\hline consumo & Consumen agua hervida & 24 & 60 \\
\hline alimentario & Consumen agua clorada. & 1 & 2.5 \\
\hline \multirow{7}{*}{$\begin{array}{l}\text { Uso de la medicina } \\
\text { tradicional y } \\
\text { científica para } \\
\text { cuidar su salud. }\end{array}$} & Usa remedios caseros para tratar a sus & 15 & 37.5 \\
\hline & hijos con diarrea. & & \\
\hline & $\begin{array}{l}\text { Cuando su hijo tiene diarrea lo lleva al } \\
\text { centro de salud. }\end{array}$ & 13 & 32.5 \\
\hline & $\begin{array}{l}\text { Cuando su hijo tiene diarrea compra } \\
\text { medicina en la farmacia. }\end{array}$ & 7 & 17.5 \\
\hline & $\begin{array}{l}\text { Usa remedios caseros para tratar a sus } \\
\text { hijos que tienen tos. }\end{array}$ & 12 & 30.0 \\
\hline & $\begin{array}{l}\text { Cuando su hijo tiene tos lo lleva al } \\
\text { centro de salud. }\end{array}$ & 13 & 32.5 \\
\hline & $\begin{array}{l}\text { Cuando su hijo tiene tos compra } \\
\text { medicina en la farmacia. }\end{array}$ & 8 & 20.0 \\
\hline
\end{tabular}

pesca y ante la transformación de las aguas y del aire por contaminación [29].

Los actores sociales del Centro Poblado Menor Dos de Mayo proponen realizar actividades con tecnología de poco impacto en el medio ambiente: "manejo de la basura y aguas servidas, organización del territorio y reforestación de las cabeceras de los cerros".

La sostenibilidad ambiental comprende acciones humanas que mantienen la capacidad de los ecosistemas para producir el rango de bienes y servicios de los cuales dependen todas las formas de vida (11).

Entonces, uno de los aspectos que debe considerar el modelo de cuidado para el desarrollo comunal es el aprovechamiento de los recursos naturales, usando estrategias para no agotarlos y conservar los ecosistemas.

Estas consideraciones en el Modelo de Cuidado permitirán diseñar políticas públicas consistentes, coherentes y generen sinergias dentro del enfoque de desarrollo humano sostenible, una ampliación de las capacidades humanas y al aumento de las oportunidades, el bienestar de las personas y generar una sociedad con mayor cohesión social en la que las personas se sientan parte de un colectivo que sea solidario con ellas, con el cual se sientan identificados y donde sus intereses sean protegidos. 


\section{Conclusiones}

El desarrollo sostenible de la Comunidad Dos de Mayo en su dimensión económica se caracteriza por baja deserción escolar de las generaciones jóvenes en los niveles primario y secundario, aumentando sus probabilidades de acceso a un trabajo mejor remunerado; las familias se dedican a la agricultura y comercio como actividad económica potencial para salir de la pobreza. Aspecto que el modelo de cuidado debe fortalecer.

El ejercicio de la ciudadanía facilita el reconocimiento de los derechos sociales, económicos, de salud en las familias al contar con su documento de identidad. El modelo debe considerar estrategias con enfoque de derechos humanos para que el ejercicio ciudadano sea efectivo y el Estado asuma su rol responsable del desarrollo humano sostenible de las comunidades rurales.

El desarrollo sostenible de la Comunidad Dos de Mayo en su dimensión social desde sus determinantes sociales intermedios fueron: adecuadas condiciones de vida por el mayor acceso a vivienda propia, servicios de agua, desagüe; las generaciones jóvenes tienen mayor oportunidad de una vida larga y saludable por haber tenido peso adecuado al nacer, frecuencia adecuada de alimentación complementaria, acceso a servicios de salud (control de gestante, inmunizaciones, crecimiento y desarrollo y consulta médica); adecuadas prácticas de actividad física: caminatas, juego con los hijos, paseos con la familia. Aspectos que el modelo de cuidado debe considerar.

El desarrollo sostenible en su dimensión ambiental evidencia prácticas ecológicas como el uso del gas para cocinar los alimentos (determinante cultural), pero la gran mayoría de las familias usan leña para cocinar, producto de la tala de árboles para realizar el cultivo en campo abierto, práctica común en la mayoría de los migrantes que provienen de la sierra.

1. El desarrollo sostenible en su dimensión cultural se caracteriza por prácticas alimentarias seguras: consumo de alimentos frecuente al día, los niños siempre ingieren su desayuno, consumen agua hervida; el uso de las plantas medicinales como capital social de conocimiento para desarrollar estrategias para la salud, el desarrollo agroindustrial, el turismo vivencial e incremento de sus recursos económicos.

\section{Referencias}

[1] J. Simonsen, Población y desarrollo sustentable, IV Reunión Nacional sobre Población (Asociación Multidisciplinaria de Investigación y Docencia en Población, Fondo de Población de Naciones Unidas, Perú, 1995), pp. 10.

[2] D. Bogado, A. Lijeron, C. Vaca, El éxodo de profesionales benianos y su impacto en el desarrollo regional (Offset Boliviana Ltda., Bolivia, La Paz, 2002), pp. 9.

[3] Instituto Nacional de Estadística e Informática, Encuesta Demográfica y de Salud Familiar Nacional y Departamental (INEI, Lima, Perú, 2011), pp. 30, 36, 44.

[4] A. Sánchez, INEl: el $74 \%$ de los peruanos vive en zonas urbanas y cada vez serán más. En Diario El Comercio, 29 de febrero del 2012.

[5] C. Polo, Resposta Social das Políticas Públicas de Saúde na Perspectiva dos Idosos. Contribuições da Enfermagem - Estudo Comparado Brasil / Perú (Universidade Federal do Rio de Janeiro, Centro de Ciências da Saúde, Escola de Enfermagem Anna Nery Coordenação Geral de Pós-Graduação e Pesquisa, Curso de Doutorado em Enfermagem Núcleo de Pesquisa Enfermagem em Saúde Coletiva, Rio de Janeiro, 2009), pp. 63-68.

[6] F. Baum, The new public health, 2nd ed. (Oxford University Press, Melbourne, 2002).

[7] A. Gramsic, Cuadernos del cárcere, 3ra ed. Vol. 3 (Civilización Brasileira, Rio de Janeiro, 2007), pp. 1891-1937.

[8] P. Guareschi, Sociologia da prática social (Vozes, Petrópolis, Rio de Janeiro, 1992).

[9] M. Lowy, Método dialético e teoria política. (Paz e Terra, Rio de Janeiro, 1989).

[10] S. Rueda, Habitabilidad y calidad de vida, 1997 (citado 20 Abril 2013), Disponible en http://habitat.aq.upm.es/cs/p2/a005.html

[11] D. Reed. Ajuste Estructural, Ambiente y Desarrollo Sostenible (Nueva Sociedad, Venezuela, 1996).

[12] Programa de las Naciones Unidas para el Desarrollo, Superar la pobreza humana, Informe sobre la pobreza 2000 (PNUD, New York, Estados Unidos, 2000).

[13] Fondo Internacional de Desarrollo Agrícola, Informe sobre la pobreza rural 2011 (FIDA, Roma; Italia, 2011), pp. 21, 22,61.

[14] Instituto Nacional de Estadística, Geografía e Informática de México, II Conteo Nacional de Población y Vivienda, Perfil Socioeconómico de los Estados Unidos Mexicanos (INEGI, México, 2008).

[15] W. Rostow, The Process of Economic Grouth, 
2nd ed. (Norton Chh XII, New York, 1962).

[16] Comisión sobre Determinantes Sociales de la Salud, Lograr la equidad en salud: desde las causas iniciales a los resultados justos, Declaración provisional (Organización Mundial de la Salud, 2005).

[17] C. Possas. Epidemiologia e sociedade. Heterogeneidade estructural no Brasil (Hucitec, Sao Paulo, 1989).

[18] Organización Panamericana de la salud. Condiciones de Salud en las Américas, Vol I (1994), pp. 549.

[19] Programa de las Naciones Unidas para el Desarrollo, Anexo: Índice de pobreza humana para países en desarrollo 1998 (citado 29 Enero 2012), Disponible en URL:htpp://es.wikipedi.org/wiki/Anexo:Índice de_pobreza_humana_para_paises:

en_desarrollo

[20] $H$. Ashworth. Infección e inmunidad en lactantes de bajo peso al nacimiento. En Nutrición, inmunidad e infección en la infancia, Resumen del 45 a Seminario de Nestlé Nutrition, 1999.

[21] M. De Curtis, C. Pieltan, J. Rigo, Nutrición en los lactantes de pretermino al momento del alta. En N. Raiha N, FF. Rubaltelli editores. Las fórmulas infantiles: más cerca del patrón de referencia, Nestlé Nutrición Workshop Series 47aㅡ Pediatric Program Suplemment (2000) 37-39.

[22] K. Tontisirin, L. Bhattacharjee. Lastre global de la desnutrición y las infecciones en la infancia. En: Nutrición, inmunidad e infección en la infancia. Resumen del $45^{\text {a }}$ Seminario de Nestlé Nutrition, 1999.

[23] L. Fernández, M. Peñuela. Crecimiento y neurodesarrollo del recién nacido de alto riesgo. Bol Med Hosp Infant Mex 56 (1999)
623-34.

[24] Oficina de Desarrollo Humano del Programa de las Naciones Unidas para el Desarrollo, Política social: capacidades y derechos, Análisis y propuestas de políticas sociales en República Dominicana, Vol I (Ministerio de Economía, Planificación y Desarrollo, Republica dominicana, 2010).

[25] A. Sen, Desarrollo y Libertad (Planeta, Barcelona, España, 2000).

[26] H. D'Orville, En Pascual Jordi, Rio +20 y cultura, Abogando por la cultura como pilar de la sostenibilidad, Agenda 21 de la Cultura (Comisión de cultura de Ciudades y Gobiernos Locales Unidos-CGLU, Barcelona, 2012).

[27] C. Lip, F. Rocabado, Determinantes sociales de la salud en Perú, Cuadernos de Promoción de la Salud № 17. Ministerio de Salud (Universidad Norbert Wiener, Organización Panamericana de la Salud, Lima, Perú, 2005).

[28] B. Kliksberg, El rol del capital social y de la cultura en el proceso de desarrollo. En B. Kliksberg y L. Tomassini (comp.), Capital social y cultura: claves estratégicas para el desarrollo (Fondo de Cultura Económica- BIDUniversidad de Maryland-Fund. Felipe Herrerra, 2000).

[29] A. Sen, Discurso inaugural ante la 52 Asamblea Mundial de la Salud (Ginebra, Suiza, 1999).

E-mail: mprq2007@hotmail.com 